\title{
Assessment of Muscle Fatigue during Isometric Contraction using Autonomic Nervous System Correlates
}

\author{
Alberto Greco*, Gaetano Valenza, Antonio Bicchi, Matteo Bianchi, and Enzo Pasquale Scilingo \\ Department of Information Engineering and Research Center "E. Piaggio", University of Pisa, Via G. Caruso 16 - 56122, Pisa, Italy. \\ *Corresponding author e-mail: alberto.greco@centropiaggio.unipi.it
}

\begin{abstract}
Muscle fatigue is a complex phenomenon that results in a reduction of the maximal voluntary force. Measuring muscle fatigue can be a challenging task that may involve the use of intramuscular electrodes (i.e., intramuscular electromyography (EMG)) or complex acquisition techniques. In this study, we propose an alternative non-invasive methodology for muscle fatigue detection relying on the analysis of two Autonomous Nervous System (ANS) correlates, i.e., the electrodermal activity (EDA) and heart rate variability (HRV) series. Based on standard surface EMG analysis, we divided 32 healthy subjects performing isometric biceps contraction into two groups: a fatigued group and a non-fatigued group.

EDA signals were analyzed using the recently proposed cvxEDA model in order to derive phasic and tonic components and extract effective features to study ANS dynamics. Furthermore, HRV series were processed to derive several features defined in the time and frequency domains able to estimate the cardiovascular autonomic regulation. A statistical comparison between the fatigued and the non-fatigued groups was performed for each ANS feature, and two EDA features, i.e., the tonic variability and the phasic response rate, showed significant differences. Moreover, a pattern recognition procedure was applied to the combined EDA-HRV feature-set to automatically discern between fatigued and non-fatigued subjects. The proposed SVM classifier, following a recursive feature elimination stage, exhibited a maximal balanced accuracy of $83.33 \%$. Our results demonstrate that muscle fatigue could be identified in a non-invasive fashion through effective EDA and HRV processing.
\end{abstract}

Keywords: Muscle fatigue, Autonomic nervous system, Heart rate variability, Electrodermal activity, Pattern recognition

\section{INTRODUCTION}

Fatigue is a complex human condition that reflects several physiological and psychological states [1,2]. Rather than being generated by a single cause [3], it depends on many central and peripheral phenomena [4] and can affect different sites of the neuromuscular system including the motor cortex, the moto-neuron, the neuromuscular transmission, the excitationcontraction coupling, or the contraction mechanisms [5]. According to the anatomical site (or sites), it is possible to distinguish among central fatigue, fatigue of the neuromuscular junction, or muscle fatigue [5].

Muscle fatigue is defined as a reduction in the ability of a muscle to generate force or power induced by a constant stimulation $[6,4]$. Its quantitative measure is challenging, also because the exact moment of muscle exhaustion is unknown [4].

By monitoring the decrease of the maximal force or power produced over time, several indices of muscular fatigue have been proposed in the literature. Particularly, previous studies showed that muscle fatigue influences the surface electromyographic signal (sEMG) in a complex way [7], with a progressive decrease in the mean or median frequencies of the power spectral density function during isometric voluntary sustained contractions $[8,9,10,11,12]$. To this end, the analysis in the time-frequency domain of sEMG can be considered as a robust alternative to the gold standard approach of the intramuscular EMG [13, 14, 15]. To monitor fatigue during a physical exercise, especially in ecological scenarios, the wearability and comfort of the measuring system is extremely relevant. However, effective wireless wearable sEMG systems currently in the market are usually expensive and present several limitations regarding comfort, encumbrance, and intrusiveness.

Several studies showed that autonomic nervous system (ANS) dynamics is directly involved in voluntary muscle contractions $[16,17]$. In detail, during such contractions both the sympathetic and parasympathetic branches of the ANS are activated through the brainstem activity $[18,16,19]$, as well as, through chemo-, mechano- and baroreceptor afferents [20, 21]. The effects of ANS modulation during fatigue are evident when referring to cardiovascular dynamics, as heartbeat, respiration rate, and blood pressure significantly changes during intense exercise [22, 23, 24, 23, 25]. Specifically, increased heart rate (HR), as well as oxygen uptake and EMG activity were observed during prolonged exercises [26, 27, 28, 29, 55, 31]. In this context, Heart rate variability (HRV) series, being modulated by sympathetic and parasympathetic interplay, was also investigated $[32,33,34]$, with the hypothesis of a sympathetic withdrawal or a vagal tone restoration occurring after maximal exercise [35]. Due to methodological limitations, previous investigations observed significant changes in HRV spectra after 
fatiguing training sessions [36, 37, 33, 38, 39], whereas few studies monitored heartbeat dynamics during the fatiguing task [40, 41, 42].

To our knowledge, other markers of ANS dynamics, such as electrodermal activity (EDA) [43, 44, 45, 46], has not been investigated in the frame of muscle fatigue induced by physical exercise. EDA is a widely used physiological signal modulated by attentional, affective, and motivational cognitive processes. EDA is directly controlled by the sympathetic branch of the ANS [47] and is used as an effective biomarker of emotional responsiveness, stress, and mental fatigue [48, 49, 50, 47].

Therefore, we hypothesize that combined HRV and EDA analyses can be profitably used to improve on the state of the art automatic recognition of the muscle fatigue condition from ANS. Contrary to the intramuscular EMG, both HRV and EDA are physiological series that can be monitored through noninvasive sensors (i.e., surface electrodes).

Accordingly, here we propose to assess muscle fatigue only using ANS measurements acquired from wearable devices, possibly opening novel applications for unobtrusive and ecological muscular fatigue monitoring. Specifically, we investigate whether parameters extracted from EDA and HRV during isometric exercise are able to statistically discriminate between a group of fatigued subjects and a control group (non-fatigue). Moreover, a pattern recognition system is propoed to automatically discern data from the two groups at a single-subject level.

\section{METHODS}

\subsection{Subject Recruitment and Experimental protocol}

Thirty-two young right-handed adults (mean age $29.25 \pm$ 3.38 (SD) - 16 males) participated in the study. Before performing the experiment, all subjects signed an informed consent to take part in the study. All experimental procedures were approved by the local ethical committee. None of the participants had a history of neurological disorders, musculoskeletal injuries, or cardiovascular diseases, or assumed antidepressants or medications that could have affected the ANS functioning and hence the experimental outcomes.

Subjects were required to perform two isometric force production tasks with their right arm: a maximum voluntary isometric contraction (MVC) and a submaximal fatiguing contraction. More in detail, the experimental paradigm consisted of three stages:

- each subject sat on a comfortable chair, with the elbow completely extended along the body, and a strap fixed on the wrist and connected to a load cell (i.e., dynamometer) [55]. To measure the MVC, the elbow flexion range of motion had to be performed completely, starting from a full extension to avoid/minimize possible compensation by the shoulders and trunk. We instructed subjects to increase the isometric force exerted by the right arm, from baseline to their maximum, and to maintain this maximum for $5 \mathrm{~s}$. All subjects were verbally encouraged to maximize their force production while their MVC was measured by the dynamometer [56, 3]. Subjects performed three MVC trials and the average of the three measures was used as the reference value to compute the target force for the submaximal fatiguing contraction. Subjects were given a rest period of 2 min after each MVC trial to minimize fatigue and ensure the correct exertion of the maximum force at each trial.

- After the MVC measurement phase, we acquired EMG, EDA and ECG data as it follows: the EMG activity measured over the biceps brachii (short and long head), upper trapezius, deltoideus, triceps brachii, brachioradialis, flexor carpi radialis, and extensor digitorum communis was recorded using surface electrodes. Surface sensors were placed in single differential mode with two contact electrodes of $10.0 \times 1.0 \mathrm{~mm}$ placed in parallel with an interelectrode distance of $10.0 \mathrm{~mm}$. This is considered the optimum interelectrode spacing for reducing crosstalk in sEM signals that contain baseline noise [57]. The two contact electrodes are assembled in a case of $41 \times 20 \times$ $5 \mathrm{~mm}$. This kind of electrode is incorporated in the commercial equipment 16-bit A/D Bagnoli Delsys EMG Systems. Following the SENIAM guidelines, the sensors were placed along the longitudinal midline of each muscle (with the long side parallel to the muscle fibers) to minimize crosstalk [58]. To determine the precise location, origin, insertion, and function of the muscle under study as well as any nearby muscles that may produce undesirable signals, the placement procedure was preformed by a clinical expert in anatomy. In addition, to ensure that highquality signals are recorded, once the sensors location was determined, subjects were asked to contract the muscle. Then, the sensor location was being slightly shifted until the detected signal was maximized. In this study, we focused only on the short-head biceps brachii (which assists with adduction), since the experimental task (equivalent to a biceps curl) allowed to maximize biceps contraction, Concerning ECG and EDA sensors, standard disposable $\mathrm{Ag} / \mathrm{AgCl}$ electrodes were used. ECG was recorded from a modified lead II configuration, which places the right electrode below the sternum and the left one on the left side of the torso below the ribcage. As suggested in [59], EDA electrodes were placed over the volar surface of the distal phalanges of the index and middle fingers of the left hand (non-dominant). A 24-bit A/D Biopac MP35 was used to acquire ECG and EDA signals. After the electrode montage, subjects were invited to comfortably sit with eyes open and their upper limbs along the body in a silent room for a 5 min resting state, while the EDA, ECG and sEMG were recorded with a sampling frequency of $2000 \mathrm{~Hz}$.

- Finally, each subject performed a submaximal fatiguing task with the elbow flexed at 90 degree, the arm along the body (see Figure 1), and a strap fixed on the right wrist and connected to a load equal to $40 \%$ of their MVC. Then, subjects were asked to maintain it as long as they could.

To avoid confounding factors possibly affecting the recorded signals, we controlled for temperature and humidity level inside 


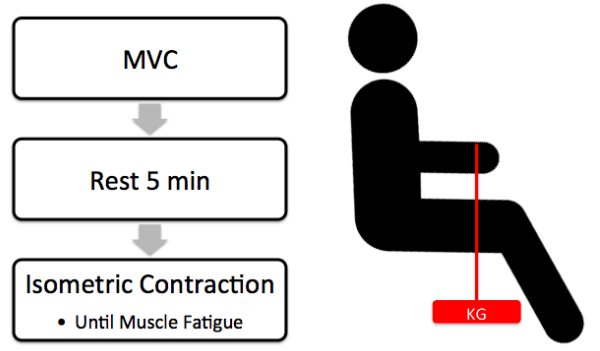

Figure 1: Experimental protocol scheme

the room. Throughout the experiment, subjects comfortably sat on a chair and were instructed to avoid speaking and strong changes in the respiration activity (such as breath hold or hyperventilation). Moreover, none of the participants were taking antidepressants or medications known to affect the ANS functioning or have had a history of neurological disorders, musculoskeletal injuries, or cardiovascular diseases. Before and after the experiment, each subject was asked to fill out psychometric tests to assess their stress level (State-Trait Anxiety Inventory test [51]), emotional state (Positive Affect and Negative Affect Scales [52]) and physical fatigue level through ad-hoc questions selected from the Multidimensional Fatigue [53] Inventory and Fatigue Assessment Scale tests [54]. The two groups of subjects did not show any statistical significant differences between the scores of all psychometric tests.

\section{2. sEMG Analysis}

Surface EMG analysis was used as reference analysis to distinguish fatigued subjects from non-fatigued ones [5]. The EMG signals of the biceps and other monitored muscles were filtered with a Infinite Impulse Response (IIR) comb notch filter $(50 \mathrm{~Hz})$. Then a zero-phase Butterworth IIR band-pass filter was used to retain frequencies between 30 and $500 \mathrm{~Hz}$. EMG spectral analysis was performed using the Discrete Fast Fourier Transform estimating the power spectra in a 1s moving Hamming window. In fact, it was proven that in case of isometric, constant force, fatiguing contractions, the EMG signal might be considered stationary for epoch durations of about $1-2$ seconds [5]. There was no overlap between consecutive time windows, according to previous findings [60]. For each 1-s time window, we calculated the median frequency value to obtain the trend of the Median Frequency (MNF) along the time. Subjects who showed a decrease in MNF over time were considered as fatigued $[5,60]$.

\subsection{EDA Processing using cvxEDA algorithm}

EDA refers to changes in the electrical properties of the skin that are directly related to the eccrine sweat gland activity. The EDA is measured by monitoring skin conductance (SC) changes over specific body sites where the concentration of the eccrine glands is high. In this study the EDA was acquired on the finger phalanxes of the non-dominant hand [47]. Due to the direct control of the sweat glands activity performed by the sympathetic branch of the ANS (and in particular the sudomotor nerve), the EDA analysis is considered one of the best ways to monitor the sudomotor nerve activity (SMNA) and, consequently, ANS activity.

It is possible to decompose the EDA signals into two components, phasic and tonic, which differ for their time scales and their relationships with the external stimuli $[61,47]$. The tonic component represents the conductance level (SCL) of the EDA signal, i.e., the slow-varying baseline level, whereas the phasic component refers to faster variations directly related to an exogenous stimulus. A single response to a stimulus is generally called skin conductance response (SCR).

The whole EDA signal results from the sum of these components, that provide relevant and non-redundant information about ANS dynamics. We extracted this information by decomposing the EDA signal through an previously validated approach: the cvxEDA model [61] (available online at:

http://www.mathworks.com/matlabcentral/fileexchange/53326cvxeda).

Here we briefly summarize the main methodological steps behind the cvxEDA model for EDA dynamics (details can be found in [61]). The model assumes EDA as the output of a linear time-invariant system to a sparse non-negative driver signal. CvxEDA describes the EDA signal ( $y$ ) as a sum of three terms: a phasic component (r), a smooth tonic component $(\mathrm{t})$, and an additive independent and identically distributed zeroaverage Gaussian noise term $(\epsilon): y=r+t+\epsilon$.

The tonic component is modeled by the sum of an offset term, a linear trend, and a smoothed cubic spline that are in accordance with physiological properties: $t=B \ell+C d$. Where $B$ is a matrix of cubic B-spline basis functions, $\ell$ is the vector of spline coefficients, $C$ is a $N \times 2$ matrix with $C_{i, 1}=1, C_{i, 2}=i / N$ (with $N=$ length of the EDA signal), and $d$ is a $2 \times 1$ vector containing the offset and slope coefficients for the linear trend.

The phasic component results from the convolution between a signal that represents the SMNA, $p$, and an impulse response shaped like a biexponential Bateman function [63]:

$$
h(\tau)=\left(e^{-\frac{\tau}{\tau_{0}}}-e^{-\frac{\tau}{\tau_{1}}}\right) u(\tau)
$$

with $\tau_{1}=2, \tau_{0}=0.7$. This function results form a bicompartmental pharmacokinetic model representing the diffusion of the sweat through the gland ducts, and can be represented, after its discrete-time approximation, in the matrix form of an autoregressive moving average model $A^{-1} M$. Where: $M$ is a tridiagonal matrix with elements $M_{i, i}=M_{i, i-2}=1, M_{i, i-1}=$ $2,3 \leq i \leq N$; and $A$ is a tridiagonal matrix with elements $A_{i, i}=\left(\tau_{1}^{-1} \delta+2\right)\left(\tau_{0}^{-1} \delta+2\right) /\left(\tau_{1}^{-1} \delta^{2}-\tau_{0}^{-1} \delta^{2}\right), A_{i, i-1}=\left(2 \tau_{1}^{-1} \tau_{0}^{-1} \delta^{2}-\right.$ $8) /\left(\tau_{1}^{-1} \delta^{2}-\tau_{0}^{-1} \delta^{2}\right), A_{i, i-2}=\left(\tau_{1}^{-1} \delta-2\right)\left(\tau_{0}^{-1} \delta-2\right) /\left(\tau_{1}^{-1} \delta^{2}-\tau_{0}^{-1} \delta^{2}\right)$, with sampling time interval $\delta$.

Using an auxiliary variable $q$ such that $q=A^{-1} p$ and $r=M q$, the final observation model results as

$$
y=M q+B \ell+C d+\epsilon .
$$

Given the EDA model (2), we aim at identifying the tonic component $(t)$ and the maximum a posteriori (MAP) neural driver SMNA $(p)$, parametrized by $[q, \ell, d]$, for the observed EDA signal $(y)$. CvxEDA rewrites the MAP problem as a constrained minimization QP convex problem: 
minimize $\frac{1}{2}\|M q+B \ell+C d-y\|_{2}^{2}+\alpha\|A q\|_{1}+\frac{\gamma}{2}\|\ell\|_{2}^{2}$

subj. to $A q \geq 0$.

Where $\alpha$ and $\gamma$ are parameters that depend on specific prior distributions (see [61]) and control the strength of the penalty (i.e., sparsity and smoothness) for the phasic and tonic components, respectively. CvxEDA finds the optimal $[q, \ell, d]$ and consequently the optimal tonic and phasic components that include the prior knowledge about the spiking sparse nature and nonnegativity of the neural signal representing the SMNA $(p)$ and the smoothness of the tonic SCL.

Table 1: List of features extracted from phasic and tonic EDA.

\begin{tabular}{|c|l|}
\hline Feature & Description \\
\hline SCRfreq & $\begin{array}{l}\text { frequency of significant SCR wrw } \\
\text { (i.e., exceeded the threshold of .5 uS [47, 62]) }\end{array}$ \\
\hline AUCphasic & Area under the curve of phasic component wrw $(\mu S s)$ \\
\hline MaxPeak & $\begin{array}{l}\text { maximum amplitude of significant peaks of } \\
\text { SMNA signal wrw }{ }^{1}(\mu S)\end{array}$ \\
\hline MeanAMP & Mean value of the SMNA signal wrw $(\mu S)$ \\
\hline STDphasic & Standard deviation of SMNA signal wrw $(\mu S$ \\
\hline STDtonic & Standard deviation of tonic signal wrw $(\mu S$ \\
\hline MeanTonic & Mean value of the tonic component wrw $(\mu S)$ \\
\hline EDAsymp & $\begin{array}{l}\text { Index of sympathetic nervous system activity }\left(\mu S^{2}\right) . \\
\text { PSD of the EDA signal within the } 0.045-0.25 \mathrm{~Hz} \text { band. }\end{array}$ \\
\hline
\end{tabular}

wrw $=$ within response window (i.e., 5 secs before the end of the task)

We then extracted several features from both the tonic and phasic signal as summarized in Table 1. In addition to the standard statistical features in the time domain, we also computed the power spectral density (PSD) of the EDA signal in the frequency band $0.045-0.25 \mathrm{~Hz}$ (i.e. EDAsymp), which has been demonstrated to be a reliable parameter for the assessment of the sympathetic nervous system activity [63].

\subsection{ECG processing}

ECG signals were band-pass filtered $(0.50-40 \mathrm{~Hz})$ to reduce noise and motion artifacts. The QRS-waveforms and each relative R-peaks were detected using the Pan-Tompkins method [64]. All time series were visually inspected to ensure that no beat was discarded. After accounting for the missed R-wave beats, the R-R interval time series (RR) were computed. Moreover, the non-evenly sampled RR interval series were interpolated $(4 \mathrm{~Hz})$ by means of a cubic spline functions, i.e. the HRV time series. A set of features in the time and frequency domain was estimated from every RR and HRV series. More in detail, we computed the mean (meanRR) and the standard deviation (stdRR) of the RR intervals within a time window, the square root of the mean squared differences of successive RR intervals (RMSSD), the number of interval differences of successive RR intervals greater than $50 \mathrm{~ms}$ divided by the total number of RR intervals (pNN50).

In the frequency domain, we defined some features for the assessment of sympathetic and parasympathetic activities. More specifically, we estimated the power spectral density of HRV signals applying the Welch's periodogram method with Blackman window and $50 \%$ of overlapped segments. The fast Fourier transform was computed for each windowed segment and the power spectra of the segments were averaged. We then calculated the power within the two main spectral bands: the Low Frequency band (LF, from $0.04 \mathrm{~Hz}$ to $0.15 \mathrm{~Hz}$ ), and the High Frequency band (HF, from $0.15 \mathrm{~Hz}$ to $0.4 \mathrm{~Hz}$ ). The HF components of HRV are known to be influenced by the parasympathetic system. In contrast, the low-frequency components (LF, $0.045-0.15 \mathrm{~Hz}$ ) are influenced by both the sympathetic and parasympathetic nervous systems. Moreover, we calculated also the ratio between the LF and the HF power was computed in order to derive information about the sympatho-vagal balance (although this has not been fully accepted as an accurate measure of the ANS balance since the LF band also contains parasympathetic dynamics) [66].

In addition, also some geometrical indexes were extracted, such as the HRV triangular index (HRVtri), and the triangular interpolation of RR interval histogram (TINN). HRVtri was computed following the standard $1 / 128$ seconds bin width, as recommended in [65].

All HRV features are listed with their relative brief description in Table 2.

Table 2: List of features extracted from HRV signals.

\begin{tabular}{|c|l|}
\hline Feature & Description \\
\hline meanRR & mean value of RR intervals $[s]$ \\
\hline stdRR & standard deviation of RR intervals $[s]$ \\
\hline pNN50 & $\begin{array}{l}\text { Number of successive RR interval pairs }>50 \mathrm{~ms} \\
\text { divided by the total number of RR intervals }[\%]\end{array}$ \\
\hline RMSSD & $\begin{array}{l}\text { Square root of the mean squared differences between } \\
\text { successive RR intervals }[s]\end{array}$ \\
\hline LF & Absolute powers of LF band $\left[s^{2}\right]$ \\
\hline LFnu & LF/(LF+HF) \\
\hline HF & Absolute powers of HF band $\left[s^{2}\right]$ \\
\hline HFnu & HF/(LF+HF) \\
\hline LF/HF & Mean value of the tonic component wrw $(\mu S)$ \\
\hline HRVtri & $\begin{array}{l}\text { The integral of the RR interval histogram divided } \\
\text { by the height of the histogram }\end{array}$ \\
\hline TINN & Baseline width of the RR interval histogram $[s]$ \\
\hline
\end{tabular}

\subsection{Statistical Analysis and Classification Procedure}

In order to detect whether subjects were fatigued or not, according to a literature review (e.g. $[5,67]$ and references therein), we divided the isometric exercise session into two subsessions: the first $25 \%$ of trial duration; the last $25 \%$ of trial duration. The EMG-MNFs computed on the first and last quarter of the experiment were statistically compared, and a subject was classified as fatigued if a significant decrease were found (see two examples of a fatigued and a non-fatigued subjects in Figure 2). According to the results of this statistical analysis, we divided the subject-set into two groups: fatigued (F) subjects and non-fatigued (N-F) subjects.

For each subject, we computed the aforementioned features within the first and the last $25 \%$ of EDA signal recorded during 
the isometric exercise, and considered the difference between these two values. Instead, given the frequency range of the HRV $(<0.4 \mathrm{~Hz})$, we could not segment the HRV signal within the first and the last $25 \%$ of the session (time windows less than $30 \mathrm{~s}$ ). Therefore, in this case the features were calculated within the whole experimental session and the obtained value were subtracted by the one calculated during the resting phase.

\subsubsection{Statistical Analysis}

For each feature, we compared values between fatigued and non-fatigued conditions using the non-parametric MannWithney $\mathrm{U}$ test under the null hypothesis that the medians of the two groups were equal. The choice of a non-parametric test was justified by the fact that most of the features were not normally distributed as demonstrated by the Shapiro Wilk test $(\mathrm{p}<0.05)$. All p-values were corrected following the Holm-Bonferroni's method.

\subsubsection{Classification Procedure}

We applied a pattern recognition algorithm to distinguish between the two groups of subjects, only using features of ANS dynamics.. The algorithm used a support vector machine classifier, which implemented a recursive feature elimination (SVMRFE) procedure $[68,69,70]$. The algorithm was validated through a leave-one-subject-out procedure (LOSO) (i.e. leaveone-out cross validation), which is a nearly unbiased estimator of the out sample error $[71,50,72]$.

In detail, within the LOSO scheme, considering $N$ subjects, iteratively we split the feature-set in a training set, comprising data from $(N-1)$ participants, and a test set comprising data from the remaining subject. Moreover, for each training set, a recursive feature elimination (RFE) algorithm is applied. We specifically chose a recently developed nonlinear SVM-RFE that employs a radial basis function kernel and includes a correlation bias reduction strategy [68]. At each iteration of the RFE, the SVM model is trained. The feature with the smallest ranking criterion is removed since it has the least effect on classification [68]. The remaining features are kept for the SVM model used at the next iteration. This process is repeated until all the features have been removed. Then the features are sorted according to their removal order. The later a feature is removed, the more important it is. We computed the median rank for each feature over all folds. Results of the sub-featureset that achieved the most accurate classification will be shown in the following section. The diagonal of the $2 \times 2$ matrix represents the percentage subjects correctly classified for each of the two classes (true positives and true negatives). In the antidiagonal, we showed the percentage of the fatigued subjects that were incorrectly marked as non-fatigued and viceversa.

\section{RESULTS}

The statistical analysis on the EMG-MNF time series of the biceps demonstrated that 12 subjects experienced fatigue of this muscle. In fact, for these subjects a significantly decreasing trend on EMG-MNF was shown between the first and last $25 \%$ of the exercise duration. The remaining 20 subjects did not shown fatigue for the biceps brachii and were labeled as nonfatigued. Same evaluation procedures, although limited to the other muscles considered in this study, showed non-fatigued conditions. In addition, a visual inspection analysis was performed to confirm a proper separation of the two groups according to the MNF trend. Figure 2 shows two MNF trends over time from a fatigued subject (lower) compared to a nonfatigued subject (upper). A decreasing MDF trend, indicating fatigue condition, is reported in red, while in blue it is shown a MDF trend related to a subject who, based on our analysis, did not experience the fatigue condition.
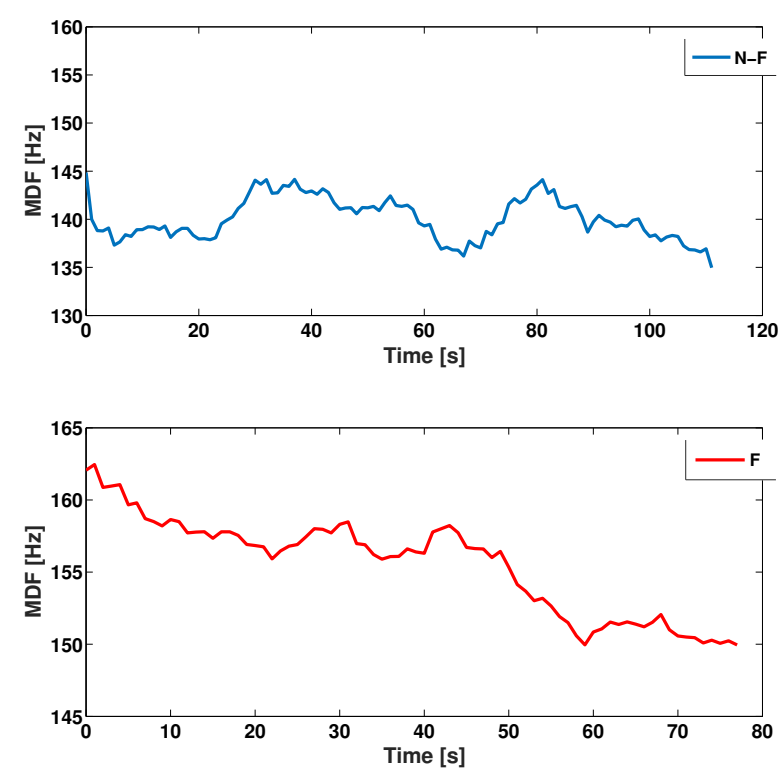

Figure 2: Example of MDF trends. In red it is reported a decreasing MDF trend related to a subject belonging to the fatigue group (F). Differently, in blue it is reported an example of a subject who did not experience the fatigue condition (N-F).

We then compared EDA and HRV features between the two groups. Concerning EDA, results are depicted in Fig. 3. Most of these features did not show significant differences. However, the standard deviation of the tonic component and the frequency of the phasic responses showed significant differences between groups.

No group-wise statistical differences were found in HRV features (see Fig. 4).

Results for the classification procedure gathered at each iteration of the RFE procedure are depicted in Figure 5. Moreover, the confusion matrix related to the best feature combination is shown in Table 3. The SVM-RFE showed that the most informative feature-subset comprised features from both the EDA and HRV series, specifically using markers of parasympathetic activity (HFnu, HF, RMSSD) and electrodermal sympathetic activity (AUCphasic, SCRfreq, MaxPeak). Of note, such a pattern recognition system was able to classify the fatigued and non-fatigued subjects with a balanced accuracy of $83.33 \%$. 

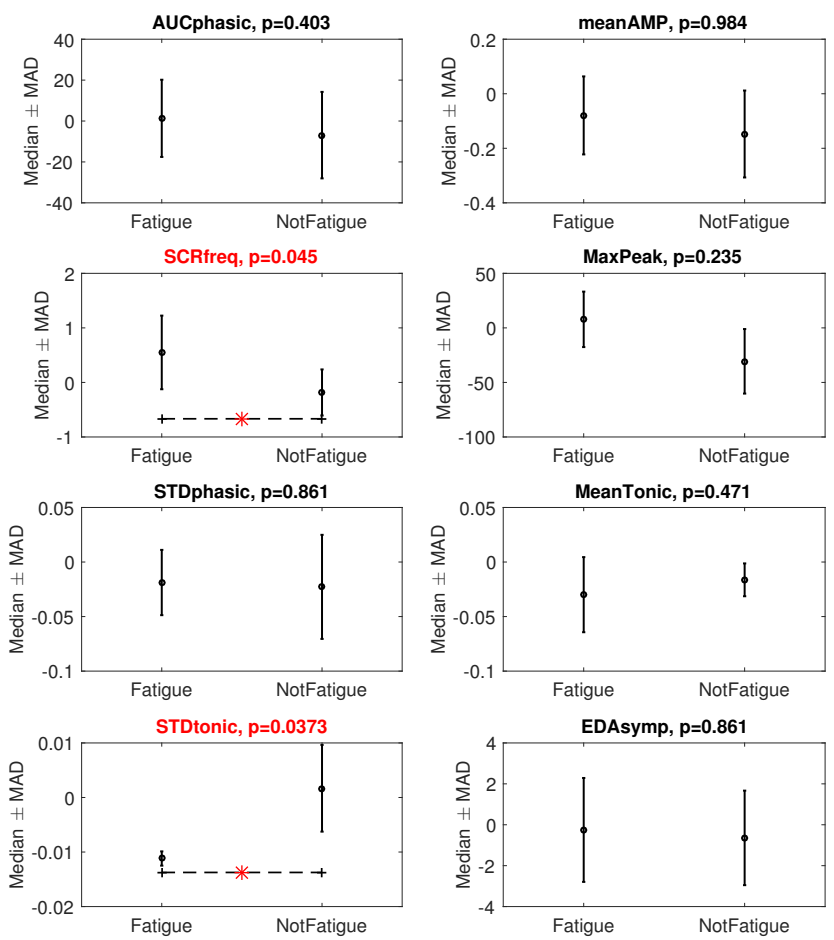

Figure 3: Statistical comparison between fatigue group and non-fatigue group. Each figure corresponds to one of the EDA features.

Table 3: Confusion matrix of Fatigued Vs Non-Fatigued groups using HRV and EDA features.

\begin{tabular}{|c|c|c|}
\hline HRV features & FATIGUE & NON-FATIGUE \\
\hline FATIGUE & $91.67 \%$ & $25.00 \%$ \\
\hline NON-FATIGUE & $8.33 \%$ & $\mathbf{7 5 . 0 0 \%}$ \\
\hline
\end{tabular}

\section{DISCUSSION and CONCLUSION}

We investigated ANS correlates of muscle fatigue through combined HRV and EDA analyses. To this end, 32 healthy subjects (16 males) were monitored during a prolonged isometric contraction. The proposed experimental task naturally induced the activation of multiple muscles of the upper limb. However, these conditions mostly led to a major involvement of the biceps brachii. By limiting our study to the monitoring of superficial electromyographic activity of the main upper limb muscles, we labeled participants as fatigued and non-fatigued according to the sEMG MNF of the biceps brachii (short and long head), upper trapezius, deltoideus, triceps brachii, brachioradialis, flexor carpi radialis, and extensor digitorum communis. To our knowledge, this is the first study proposing an automatic muscle fatigue recognition at a single-subject level based on electrodermal and cardiovascular dynamics. Indeed, our results demonstrate that it is possible to recognize muscle fatigue condition of biceps using a proper combination of EDA phasic features and linear/spectral quantifiers of cardiovascular variability.

From a methodological viewpoint, we employed a recently
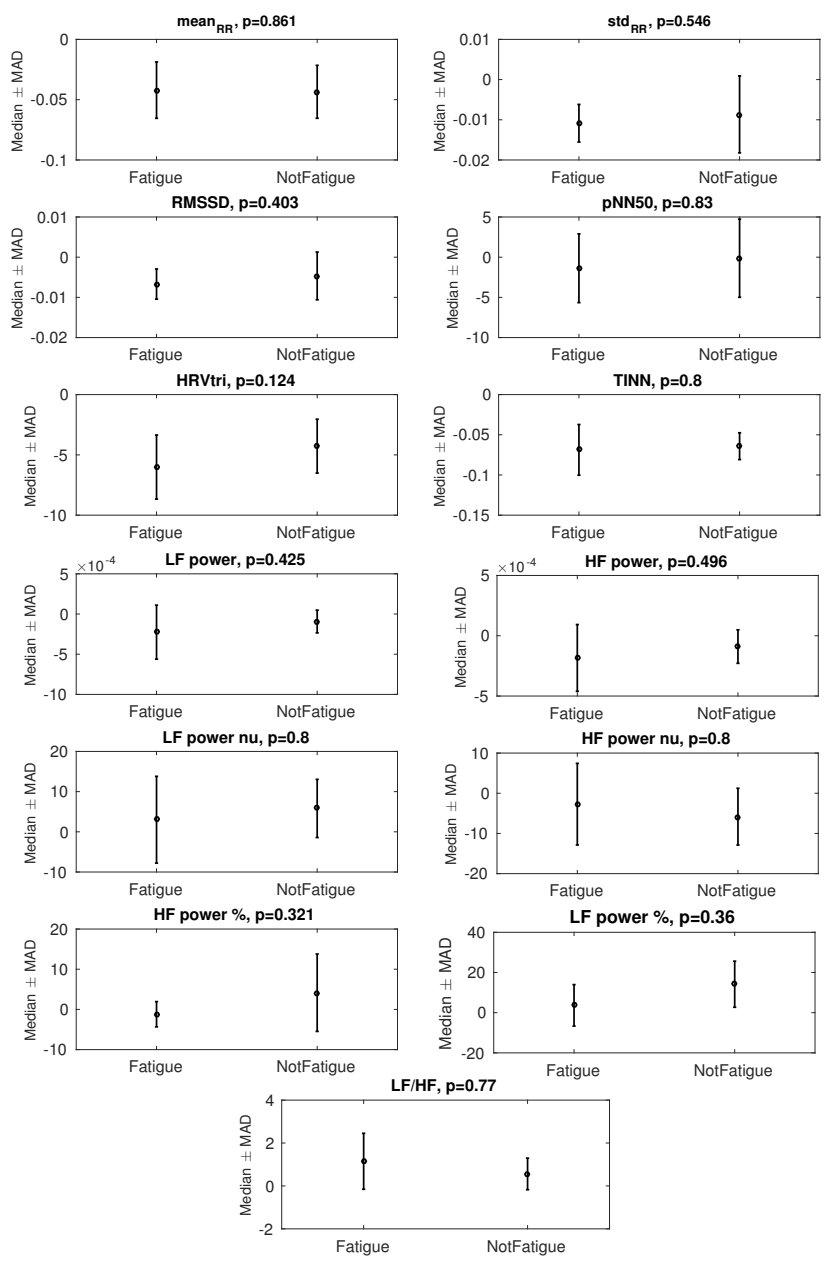

Figure 4: Statistical comparison between fatigued group and non-fatigued group. Each figure corresponds to one of the HRV features.

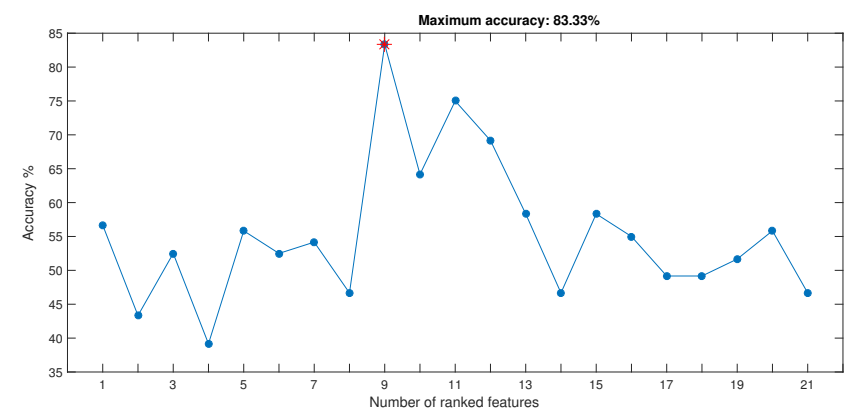

Figure 5: Statistical comparison between fatigued and non-fatigued groups. Each figure corresponds to one of the EDA features.

proposed model of EDA in order to efficiently decompose the signal into its phasic and tonic components. This processing represents an essential step to effectively derive psychophysiological markers [61, 74], In addition, markers of sympathovagal control were derived from HRV analysis performed 
in the time and frequency domains. In this way, we collected features from two of the most widely used ANS correlates, carrying complementary information.

As a preliminary, exploratory step, we performed a statistical analysis to compare ANS features between the first and last quarter of the fatiguing exercise. Features from cardiovascular variability did not show significant changes. Of note, the inability to calculate the HRV features within a smaller time window, during the exercise, may have limited our possibility to detect possible changes of the sympathovagal dynamics. On the other hand, EDA measurements revealed a significant decrease of the sudomotor nerve spike frequency, as well as an increase of the tonic variability in the case of fatigue. Previous studies report that the vagal activity is predominant during the first part of an isometric exercise, whereas the sympathetic effect prevails close to the completion of the exercise [56]. As EDA neural control is exclusively sympathetic [47, 74], we can assume that the sympathetic activity, when muscle fatigue occurs, is reflected in a more variable tonic signal (slow variations) together with a decrease of faster phasic variations.

We have also proposed a pattern recognition approach able to automatically recognize muscle fatigue at a single-subject level using only ANS correlates. In addition we demonstrated that a proper combination of HRV and EDA features significantly improves balanced classification accuracy. The automatic muscle fatigue classification was performed using nu-SVM, which was validated by means of a LOSO cross-validation. Results showed a satisfactory balanced accuracy of $83.33 \%$ when a specific feature combination was taken into account (see Table 3 ). This accuracy results from the combination of a specificity of $91.67 \%$, and a sensitivity of $75.00 \%$. Our results demonstrate that the discrimination of ANS states associated with muscle fatigue can be supported by a multivariate machine learning analysis implemented at a single-subject level, rather than using group-wise statistics. Of note, the accuracy of the non-fatigued class, i.e., $75.00 \%$, was fairly lower than the fatigue class. This could be explained by the conservative technique used to label the subjects according to a statistical analysis on sEMG spectral features. More in detail, four out of the five subjects who were misclassified in the non-fatigued group (i.e., the $25 \%$ of false positives) showed a decrease in the MNF trend from the first to the last quarter of the task. However, paired statistical analyses on such trends did not show significant differences, possibly due to the high inter-subject variability. At a speculation level, this may suggest possible muscle fatigue also in subjects belonging to the non-fatigued group. In particular, misclassified subjects may be the most fatigued ones within the nonfatigued group. Furthermore, a rigorous SVM-RFE procedure revealed that the most informative features are strongly correlated with the parasympathetic branch of cardiovascular neural control and the sympathetic activation of the electrodermal processes. Of note, the implemented SVM-RFE uses a radial basis function kernel and reduces bias due to highly correlated features. Our results also suggest that the muscle fatigue process is modulated by both the ANS branches. In this sense, EDA, which is controlled only by the sympathetic nervous system, and the HF spectral information of the HRV signal, which is strongly associated with the parasympathetic activity, have been confirmed to be an ideal way to monitor the ANS dynamics.

To summarize, this work proposes a non-invasive methodology for muscular fatigue detection. However, we are aware the non-invasiveness and simplicity of our approach comes with limitations. First, sEMGs cannot consider the effect of deeper muscles that may contribute to the task and got fatigued, and sometimes it could possibly lead to controversial outcomes, e.g. the compensation of MNF decrease in submaximal task due to the recruitment of new motor units. These issues could be addressed relying on invasive procedures or through EMG array-based acquisition and processing techniques, which can in principle identify the discharge of multi-channel action potentials by individual muscle units [73]. This would certainly improve the reliability of the results but, on the other side, it would be in contrast with the minimalistic approach that drove our work, under a sensing and/or computational point of view. A comparison between these different approaches is interesting, and it will deserve future analyses to identify the right trade-off between effectiveness and usability/simplicity.

These results pave the path towards unobtrusive and wearable devices to assess fatigue in working environments or during rehabilitative procedures in an ecological fashion. Indeed, the devices that are able to monitor, for example, HRV series are already very popular in the everyday life (e.g., smartphones and smartwatches). In addition, ANS measurements could be also used to infer the emotional state of the subjects [74, 75, 46], which can play a crucial role in the rehabilitation procedures [76].

Future endeavors will be directed to study EMG dynamics from several muscles locations, and to implement our approach into a portable device, which integrates, e.g., into a smartwatch, HRV and EDA sensors (i.e., Empatica wearable systems [77]).

\section{Acknowledgment}

The research leading to these results has received partial funding from the EU H2020 project "SoftPro: Synergy-based Open-source Foundations and Technologies for Prosthetics and RehabilitatiOn" (H2020-ICT-688857).

\section{REFERENCES}

[1] M. M. Bundele and R. Banerjee, "An svm classifier for fatigue-detection using skin conductance for use in the bits-lifeguard wearable computing system," in Emerging Trends in Engineering and Technology (ICETET), 2009 2nd International Conference on. IEEE, 2009, pp. 934-939.

[2] P. Bhat and A. Gupta, "A novel approach to detect localized muscle fatigue during isometric exercises," in Wearable and Implantable Body Sensor Networks (BSN), 2016 IEEE 13th International Conference on. IEEE, 2016, pp. 224-229.

[3] S. P. Cairns, A. J. Knicker, M. W. Thompson, and G. Sjøgaard, "Evaluation of models used to study neuromuscular fatigue," Exercise and sport sciences reviews, vol. 33, no. 1, pp. 9-16, 2005.

[4] R. M. Enoka and J. Duchateau, "Muscle fatigue: what, why and how it influences muscle function," The Journal of physiology, vol. 586, no. 1, pp. 11-23, 2008.

[5] R. Merletti and P. A. Parker, Electromyography: physiology, engineering, and non-invasive applications. John Wiley \& Sons, 2004, vol. 11. 
[6] S. Gandevia, "Spinal and supraspinal factors in human muscle fatigue," Physiological reviews, vol. 81, no. 4, pp. 1725-1789, 2001.

[7] T. G. Sandercock, J. A. Faulkner, J. W. Albers, and P. H. Abbrecht, "Single motor unit and fiber action potentials during fatigue," Journal of Applied Physiology, vol. 58, no. 4, pp. 1073-1079, 1985

[8] H. Piper, "Elektrophysiologie menschlicher muskeln springer verlag,' 1912.

[9] D. B. Chaffin, "Localized muscle fatigue-definition and measurement." Journal of Occupational and Environmental Medicine, vol. 15, no. 4, pp. 346-354, 1973.

[10] C. De Luca, "Myoelectric manifestation of localized muscular fatigue in humans," in IEEE Transactions On Biomedical Engineering, vol. 30, no. 8. IEEE-INST Electrical Electronics Engineers Inc 445 Hoes Lane, Piscataway, NJ 08855 USA, 1983, pp. 531-531.

[11] W. Li and K. Sakamoto, "The influence of location of electrode on muscle fiber conduction velocity and emg power spectrum during voluntary isometric contraction measured with surface array electrodes," Applied Human Science, vol. 15, no. 1, pp. 25-32, 2001

[12] L. Lindstrom, "Muscular fatigue and action potential conduction velocity changes studied with frequency analysis of emg signals." Electromyogr Clin Neurophysiol, vol. 10, pp. 341-356, 1970.

[13] K. Ito and Y. Hotta, "Surface electromyogram-based detection of muscle fatigue during cyclic dynamic contraction under blood flow restriction,' in Engineering in Medicine and Biology Society (EMBC), 2014 36th Annual International Conference of the IEEE. IEEE, 2014, pp. 22052208.

[14] R. H. Chowdhury, M. B. Reaz, M. A. B. M. Ali, A. A. Bakar, K. Chellappan, and T. G. Chang, "Surface electromyography signal processing and classification techniques," Sensors, vol. 13, no. 9, pp. 12431-12466, 2013.

[15] D. MacIsaac, P. Parker, and K. Englehart, "A novel approach to localized muscle fatigue assessment," in Engineering in Medicine and Biology Society, 2003. Proceedings of the 25th Annual International Conference of the IEEE, vol. 3. IEEE, 2003, pp. 2487-2490.

[16] D. B. Friedman, C. Peel, and J. H. Mitchell, "Cardiovascular responses to voluntary and nonvoluntary static exercise in humans," Journal of Applied Physiology, vol. 73, no. 5, pp. 1982-1985, 1992.

[17] J. Mitchell, D. Reeves, H. Rogers, and N. Secher, "Epidural anaesthesia and cardiovascular responses to static exercise in man." The Journal of Physiology, vol. 417, no. 1, pp. 13-24, 1989.

[18] G. Goodwin, D. McCloskey, and J. Mitchell, "Cardiovascular and respiratory responses to changes in central command during isometric exercise at constant muscle tension," The Journal of Physiology, vol. 226, no. 1, pp. 173-190, 1972.

[19] F. Iellamo, R. Hughson, F. Castrucci, J. Legramante, G. Raimondi, G. Peruzzi, and G. Tallarida, "Evaluation of spontaneous baroreflex modulation of sinus node during isometric exercise in healthy humans," American Journal of Physiology-Heart and Circulatory Physiology, vol. 267, no. 3, pp. H994-H1001, 1994.

[20] L. B. Rowell and D. S. O'Leary, "Reflex control of the circulation during exercise: chemoreflexes and mechanoreflexes," Journal of Applied Physiology, vol. 69, no. 2, pp. 407-418, 1990.

[21] F. Iellamo, J. Legramante, G. Raimondi, and G. Peruzzi, "Baroreflex control of sinus node during dynamic exercise in humans: effects of central command and muscle reflexes," American Journal of PhysiologyHeart and Circulatory Physiology, vol. 272, no. 3, pp. H1157-H1164, 1997.

[22] L. Sheldahl, L. Wann, P. S. Clifford, F. Tristani, L. Wolf, and J. Kalbfleisch, "Effect of central hypervolemia on cardiac performance during exercise," Journal of Applied Physiology, vol. 57, no. 6, pp. 1662-1667, 1984.

[23] J. Mitchell, F. Payne, B. Saltin, and B. Schibye, "The role of muscle mass in the cardiovascular response to static contractions," The Journal of Physiology, vol. 309, p. 45, 1980.

[24] A. S. Leicht, W. H. Sinclair, and W. L. Spinks, "Effect of exercise mode on heart rate variability during steady state exercise," European journal of applied physiology, vol. 102, no. 2, pp. 195-204, 2008.

[25] M. Weippert, M. Behrens, R. Gonschorek, S. Bruhn, and K. Behrens, "Muscular contraction mode differently affects autonomic control during heart rate matched exercise," Frontiers in physiology, vol. 6, 2015

[26] R. Lepers, C. Hausswirth, N. Maffiuletti, J. Brisswalter, and
J. Van Hoecke, "Evidence of neuromuscular fatigue after prolonged cycling exercise," Medicine and science in sports and exercise, vol. 32, no. 11 , pp. 1880-1886, 2000.

[27] L. Sheldahl, L. Wann, P. Clifford, F. Tristani, L. Wolf, and J. Kalbfleisch, "Effect of central hypervolemia on cardiac performance during exercise," Journal of Applied Physiology, vol. 57, no. 6, pp. 1662-1667, 1984.

[28] J. A. Schwane, S. R. Johnson, C. B. Vandenakker, and R. B. Armstrong, "Delayed-onset muscular soreness and plasma cpk and ldh activities after downhill running." Medicine and science in sports and exercise, vol. 15 , no. 1 , pp. $51-56,1982$.

[29] T. L. Hooper, D. M. Dunn, J. E. Props, B. A. Bruce, S. F. Sawyer, and J. A. Daniel, "The effects of graded forward and backward walking on heart rate and oxygen consumption," Journal of Orthopaedic Eamp; Sports Physical Therapy, vol. 34, no. 2, pp. 65-71, 2004.

[30] A. S. Oliveira and M. Gonçalves, "Positioning during resistance elbow flexor exercise affects electromyographic activity, heart rate, and perceived exertion," The Journal of Strength Eamp; Conditioning Research, vol. 23 , no. 3, pp. 854-862, 2009.

[31] C. J. Mottram, J. M. Jakobi, J. G. Semmler, and R. M. Enoka, "Motorunit activity differs with load type during a fatiguing contraction," Journal of neurophysiology, vol. 93, no. 3, pp. 1381-1392, 2005.

[32] C. R. Bellenger, L. Karavirta, R. L. Thomson, E. Y. Robertson, K. Davison, and J. D. Buckley, "Contextualizing parasympathetic hyperactivity in functionally overreached athletes with perceptions of training tolerance," International journal of sports physiology and performance, vol. 11, no. 5, pp. 685-692, 2016

[33] B. Makivić, M. Djordjević Nikić, and M. S. Willis, "Heart rate variability (hrv) as a tool for diagnostic and monitoring performance in sport and physical activities," Journal of Exercise Physiologyonline, vol. 16, no. 3, pp. 100-109, 2013.

[34] L. Wallace, K. Slattery, and A. J. Coutts, "A comparison of methods for quantifying training load: relationships between modelled and actual training responses," European journal of applied physiology, vol. 114, no. 1, pp. 11-20, 2014.

[35] T. P. Oliveira, R. Alvarenga Mattos, R. B. F. Silva, R. A. Rezende, and J. R. P. Lima, "Absence of parasympathetic reactivation after maximal exercise," Clinical physiology and functional imaging, vol. 33, no. 2, pp. 143-149, 2013.

[36] G. Vernillo, L. Agnello, A. Barbuti, S. Di Meco, G. Lombardi, G. Merati, and A. La Torre, "Postexercise autonomic function after repeated-sprints training," European journal of applied physiology, vol. 115, no. 11, pp. 2445-2455, 2015.

[37] R. K. Mehta and M. J. Agnew, "Influence of mental workload on muscle endurance, fatigue, and recovery during intermittent static work," $E u$ ropean journal of applied physiology, vol. 112, no. 8, pp. 2891-2902, 2012.

[38] C. Scott, F. Wyatt, J. Winchester, K. Williamson, A. Welter, and S. Brown, "Physiological breakpoints and maximal steady-state of cycling," Journal of Exercise Physiology Online, vol. 18, no. 3, pp. 33-46, 2015.

[39] J.-L. Chen, D.-P. Yeh, J.-P. Lee, C.-Y. Chen, C.-Y. Huang, S.-D. Lee, C.-C. Chen, T. B. Kuo, C.-L. Kao, and C.-H. Kuo, "Parasympathetic nervous activity mirrors recovery status in weightlifting performance after training," The Journal of Strength $\mathcal{E}$ Conditioning Research, vol. 25, no. 6 , pp. 1546-1552, 2011.

[40] R. Mehta, "Impacts of obesity and stress on neuromuscular fatigue development and associated heart rate variability," International Journal of Obesity, vol. 39, no. 2, pp. 208-213, 2015.

[41] T. Kiryu, T. Abe, T. Gocho, Y. Ushiyama, and M. Okada, "Snapshot evaluation of fatigue during skiing exercise," in Engineering in Medicine and Biology Society, 1998. Proceedings of the 20th Annual International Conference of the IEEE, vol. 5. IEEE, 1998, pp. 2775-2778.

[42] J. S. Petrofsky and M. Laymon, "Blood pressure and heart rate responses during a fatiguing isometric exercise in paraplegic men with hypertension," European journal of applied physiology, vol. 83, no. 4, pp. 274282, 2000.

[43] R. Calvo and S. D'Mello, "Affect detection: An interdisciplinary review of models, methods, and their applications," Affective Computing, IEEE Transactions on, vol. 1, no. 1, pp. 18-37, 2010.

[44] A. Lanatà, G. Valenza, A. Greco, C. Gentili, R. Bartolozzi, F. Bucchi, 
F. Frendo, and E. P. Scilingo, "How the autonomic nervous system and driving style change with incremental stressing conditions during simulated driving," Intelligent Transportation Systems, IEEE Transactions on, vol. 16, no. 3, pp. 1505-1517, 2015.

[45] A. Greco, A. Lanata, L. Citi, N. Vanello, G. Valenza, and E. P. Scilingo, "Skin admittance measurement for emotion recognition: A study over frequency sweep," Electronics, vol. 5, no. 3, p. 46, 2016.

[46] A. Greco, G. Valenza, M. Nardelli, M. Bianchi, L. Citi, and E. P. Scilingo, "Force-velocity assessment of caress-like stimuli through the electrodermal activity processing: Advantages of a convex optimization approach," IEEE Transactions on Human-Machine Systems, vol. 47, no. 1, pp. 91-100, 2017.

[47] W. Boucsein, Electrodermal activity. Springer, 2012.

[48] A. Greco, G. Valenza, A. Lanata, G. Rota, and E. P. Scilingo, "Electrodermal activity in bipolar patients during affective elicitation," IEEE Journal of Biomedical and Health Informatics, vol. 18, no. 6, pp. 18651873, 2014.

[49] A. Greco, G. Valenza, M. Nardelli, M. Bianchi, A. Lanata, and E. P. Scilingo, "Electrodermal activity analysis during affective haptic elicitation," in Engineering in Medicine and Biology Society (EMBC), 2015 37th Annual International Conference of the IEEE. IEEE, 2015, pp. 5777-5780.

[50] A. Greco, G. Valenza, L. Citi, and E. P. Scilingo, "Arousal and valence recognition of affective sounds based on electrodermal activity," IEEE Sensors Journal, vol. 17, no. 3, pp. 716-725, 2017.

[51] C. D. Spielberger, F. Gonzalez-Reigosa, A. Martinez-Urrutia, L. F. Natalicio, and D. S. Natalicio, "The state-trait anxiety inventory," $R e$ vista Interamericana de Psicologia/Interamerican Journal of Psychology, vol. 5, no. 3 \& 4, 2017.

[52] D. Watson, L. A. Clark, and A. Tellegen, "Development and validation of brief measures of positive and negative affect: the panas scales." Journal of personality and social psychology, vol. 54, no. 6, p. 1063, 1988.

[53] E. Smets, B. Garssen, B. d. Bonke, and J. De Haes, "The multidimensional fatigue inventory (mfi) psychometric qualities of an instrument to assess fatigue," Journal of psychosomatic research, vol. 39, no. 3, pp. 315-325, 1995.

[54] H. J. Michielsen, J. De Vries, and G. L. Van Heck, "Psychometric qualities of a brief self-rated fatigue measure: The fatigue assessment scale," Journal of psychosomatic research, vol. 54, no. 4, pp. 345-352, 2003.

[55] A. S. Oliveira and M. Gonçalves, "Positioning during resistance elbow flexor exercise affects electromyographic activity, heart rate, and perceived exertion," The Journal of Strength $\mathcal{E}$ Conditioning Research, vol. 23, no. 3, pp. 854-862, 2009.

[56] B. Maciel, L. Gallo, J. Marin Neto, and L. Martins, "Autonomic nervous control of the heart rate during isometric exercise in normal man,' Pflügers Archiv European Journal of Physiology, vol. 408, no. 2, pp. 173-177, 1987.

[57] C. J. De Luca, M. Kuznetsov, L. D. Gilmore, and S. H. Roy "Interelectrode spacing of surface EMG sensors: reduction of crosstalk contamination during voluntary contractions," Journal of biomechanics, vol. 45, no. 3, pp. 555-561, 2012.

[58] H. J. Hermens, B. Freriks, R. Merletti, D. Stegeman, J. Blok, G. Rau, C. Disselhorst-Klug, and G. Hägg, "European recommendations for surface electromyography," Roessingh research and development, vol. 8, no. 2, pp. 13-54, 1999 .

[59] M. E. Dawson, A. M. Schell, and D. L. Filion, "The electrodermal system," Handbook of psychophysiology, vol. 2, pp. 200-223, 2007.

[60] D. Farina and R. Merletti, "Comparison of algorithms for estimation of EMG variables during voluntary isometric contractions," Journal of Electromyography and Kinesiology, vol. 10, no. 5, pp. 337-349, 2000.

[61] A. Greco, G. Valenza, A. Lanata, E. P. Scilingo, and L. Citi, "Cvxeda: A convex optimization approach to electrodermal activity processing," IEEE Transactions on Biomedical Engineering, vol. 63, no. 4, pp. 797804, 2016.

[62] M. Benedek and C. Kaernbach, "A continuous measure of phasic electrodermal activity," Journal of neuroscience methods, vol. 190, no. 1, pp. 80-91, 2010.

[63] H. F. Posada-Quintero, J. P. Florian, A. D. Orjuela-Cañón, T. AljamaCorrales, S. Charleston-Villalobos, and K. H. Chon, "Power spectral density analysis of electrodermal activity for sympathetic function assessment," Annals of biomedical engineering, vol. 44, no. 10, pp. 3124
3135, 2016.

[64] J. Pan and W. J. Tompkins, "A real-time qrs detection algorithm," IEEE transactions on biomedical engineering, no. 3, pp. 230-236, 1985.

[65] T. F. of the European Society of Cardiology et al., "Heart rate variability: standards of measurements, physiological interpretation and clinical use." Circulation, vol. 93, pp. 1043-1065, 1996.

[66] G. A. Reyes del Paso, W. Langewitz, L. J. Mulder, A. Roon, and S. Duschek, "The utility of low frequency heart rate variability as an index of sympathetic cardiac tone: a review with emphasis on a reanalysis of previous studies," Psychophysiology, vol. 50, no. 5, pp. 477-487, 2013.

[67] A. Danna-Dos Santos, B. Poston, M. Jesunathadas, L. R. Bobich, T. M Hamm, and M. Santello, "Influence of fatigue on hand muscle coordination and emg-emg coherence during three-digit grasping," Journal of neurophysiology, vol. 104, no. 6, pp. 3576-3587, 2010.

[68] K. Yan and D. Zhang, "Feature selection and analysis on correlated gas sensor data with recursive feature elimination," Sensors and Actuators B: Chemical, vol. 212, pp. 353-363, 2015.

[69] I. Guyon, J. Weston, S. Barnhill, and V. Vapnik, "Gene selection for cancer classification using support vector machines," Machine learning, vol. 46, no. 1, pp. 389-422, 2002.

[70] A. Rakotomamonjy, "Variable selection using svm-based criteria," Journal of machine learning research, vol. 3, no. Mar, pp. 1357-1370, 2003.

[71] Z. Shao and M. J. Er, "Efficient leave-one-out cross-validation-based regularized extreme learning machine," Neurocomputing, vol. 194, pp. 260-270, 2016.

[72] G. C. Cawley, "Leave-one-out cross-validation based model selection criteria for weighted ls-svms," in Neural Networks, 2006. IJCNN'06. International Joint Conference on. IEEE, 2006, pp. 1661-1668.

[73] D. Farina, Y. Ken, T. Stieglitz and K. P. Koch, "Multichannel thin-film electrode for intramuscular electromyographic recordings," Journal of Applied Physiology, vol. 104, no. 3, pp. 821-827, 2008.

[74] A. Greco, G. Valenza, L. Citi, and E. P. Scilingo, "Arousal and valence recognition of affective sounds based on electrodermal activity," IEEE Sensors Journal, 2016.

[75] K. H. Kim, S. W. Bang, and S. R. Kim, "Emotion recognition system using short-term monitoring of physiological signals," Medical and biological engineering and computing, vol. 42, no. 3, pp. 419-427, 2004.

[76] M. Mihelj, D. Novak, and M. Munih, "Emotion-aware system for upper extremity rehabilitation," in Virtual Rehabilitation International Conference, 2009. IEEE, 2009, pp. 160-165.

[77] Empatica: Human data in real time, "https://www.empatica.com/", 